\title{
The Fourth and Fifth Spectra of Vanadium (V IV and V V)
}

\author{
Laura Iglesias*
}

(April 26, 1968)

\begin{abstract}
The V IV spectrum has been extended by using as light sources a condensed spark and a hollow cathode discharge. With the new data, the experimental interpretation of levels of the $3 d^{2}, 3 d 4 d$, $3 d 5 s, 3 d 5 p, 3 d 4 f, 3 d 5 d$ and $3 d 6 s$ configurations has been completed with the exception of three levels of the $3 d 4 f$ and $3 d 5 d$ configurations. Four levels of the $3 d 5 g$ electron configuration have also been found. These levels account for 340 of the 360 lines assigned to V IV in the region $675 \AA-5940 \AA$.

Three members of the $3 d n s$ series give an ionization potential of $376730 \pm 40 \mathrm{~cm}^{-1}=46.70$ volts.

The $4 d^{2} \mathrm{D}$ term of $\mathrm{V} \mathrm{v}$ has also been located in the course of this work. With the aid of the new observations between $675 \AA$ and $2200 \AA$ the value of some levels already known has been improved.
\end{abstract}

Key Words: Atomic spectra, V IV and V v; classified lines, V IV and V V spectra; spectra, V IV and V V; terms, V IV and V V spectra; vanadium, the tourth and fifth spectra of.

\section{Introduction}

The fourth spectrum of vanadium was analyzed by White $[1]^{1}$ in 1929 , who found the terms of the main configurations, $3 d^{2}, 3 d 4 s, 3 d 4 p$, and two terms of the $3 d 4 d$ configuration. From a study of related spectra, B. Edlén deduced (see A.E.L. [2]), that the singlet term ${ }^{1} \mathrm{~S}$ of the $3 d^{2}$ configuration was incorrect and that the energy level values of all singlet terms should be decreased by $698 \mathrm{~cm}^{-1}$.

As a result of new observations a complete revision and extension of the V IV spectrum has been made, and the results are presented in this paper, together with some new levels of $\mathrm{V} v$.

\section{Experimental Details}

The analyses are based on two sets of spectrograms. The first set was obtained by the author during a stay at Princeton University, and covers the region 675 to $2200 \AA$. The plates were taken with the 2 meter-grating spectrograph at Palmer Physical Laboratory, by using as light source a condensed spark in helium, under the same conditions used by A. G. Shenstone to excite Ni III [3]. In the present case V I, V II, V III, V IV and V V were excited; by observation of the polarity of the lines it was not difficult to differentiate among the lines corresponding to the various stages of ionization. (See fig. 1.)

The second set of plates, from 2200 to $5940 \AA$, was obtained by Velasco in Lund in 1953. The light source

\footnotetext{
*An invited paper. Present address: Instituto de Optica, Madrid 6, Spain.

${ }^{1}$ Figures in brackets indicate the literature references at the end of this paper.
}

was a pulsed hollow cathode discharge used under the conditions described in a paper on V III [4]; the spectral instruments used were a 21 -ft grating spectrograph in a Wadsworth mounting and a quartz prism spectrograph Hilger-E478. More than one thousand lines were then attributed to $\mathrm{V}$ III but it was pointed out that some of them might belong to V IV. In most of the grating spectrograms, it was impossible to distinguish V III from V IV lines. Other spectrograms obtained with the prism spectrograph permitted, however, an unambiguous distinction between $\mathrm{V}$ III and V IV lines. The analysis of V IV could thus be substantially extended.

On the vacuum spectrograph the plate factor was about $4.2 \AA / \mathrm{mm}$ in the first order. From 675 to $800 \AA$ all but the weakest lines were measured in the second order. In the hollow cathode spectrograms the plate factor varied from $2.0 \AA / \mathrm{mm}$ in the prism spectrograms to about $5 \AA / \mathrm{mm}$ in those taken in the first order.

Most of the wavelength values given in table 3 are averages of measurements made on two or more plates. To distinguish between the accuracy of different measurements three decimal places have been retained for the lines with an estimated probable error not higher than $\pm 0.01 \AA$. When only two decimal places are given in the wavelength, the error may range from 0.01 to $0.03 \AA$, depending on the character of the line. Correspondingly the number of significant figures in the wave number column (table 3 ) has been adjusted to the assumed accuracy of the wavelengths. The symbols used in the "Intensity" column in table 3 are those recommended by the Joint Commission for Spectroscopy [5].

About 360 lines in all were assigned to V IV. 

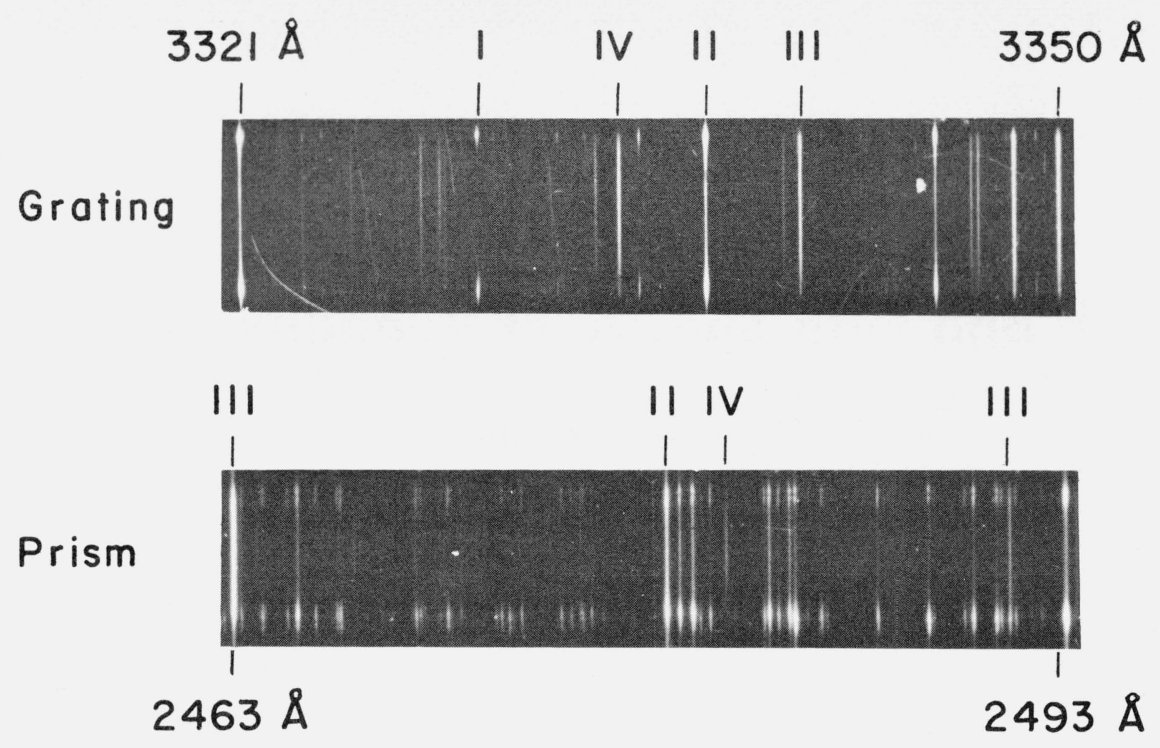

Figure 1. Hollow Cathode Spectrà of Vanadium. The lines belonging to each spectrum are indicated by the corresponding roman numeral.

On the prism spectrum V IV lines are clearly shorter and wider than V III lines of similar intensity.

\section{Analysis}

Within the main configurations only the term $3 d^{2}{ }^{1} \mathrm{~S}$ was missing. The only remaining unclassified line in the region of the $3 d^{2}-3 d 4 p$ transitions, $\lambda=884.146 \AA$, was, therefore, designated as the ${ }^{1} \mathrm{~S}^{-1} \mathrm{P}^{\circ}$ combination, giving a value of $42462.1 \mathrm{~cm}^{-1}$ for the ${ }^{1} \mathrm{~S}$ level. New transitions between the even and odd levels confirm the reality of the energy levels given in A.E.L. [2], with exception of the $3 d\left({ }^{2} \mathrm{D}\right) 4 d^{3} \mathbf{F}$ term.

With the present observations the values of the energy levels already known have been improved. An asterisk in tables 1 and 2 indicates that a slight change in the level value has been made as a result of this revision.

The determination of levels and their grouping into terms in the higher configurations did not offer special difficulties. The search for new energy levels has been made by the usual method of recurring differences. The assignment of levels to terms and electron configurations, takes into account the relative intensities of lines in multiplets, the intervals, and the relative positions of terms and configurations deduced from comparisons with such related spectra as Ti III and Ti IV. In the case of the $4 f$ electron configuration the theoretical calculations for Fe VII by Y. Shadmi were used as a guide [6].
The limit of the $3 d n x$ configurations being a ${ }^{2} \mathrm{D}$ term of $\mathrm{V} \mathrm{V}$ and the coupling nearly $L S$ for low values of " $x$ ", the designation of the levels was rather straightforward. In the case of the $4 f$ electron configuration there are a few levels that share the same $L S$ character. The designation is, therefore, not so conclusive, and the assignment has been made by comparison with Ti III [7] and Fe VII $[8,6]$, as stated above. The $3 d 5 g$ configuration is expected to show a high percentage of pair coupling. Only four levels have been found with certainty, which, accordingly, are presented in table 1 with pair-coupling notation.

As a result of this analysis, all the terms belonging to $3 d 4 d, 3 d 5 s, 3 d 5 p, 3 d 4 f, 3 d 5 d$ and $3 d 6 s$ have been found with the exception of three levels of the $3 d 4 f$ and $3 d 5 d$ configurations (tables 1 and 2). The total number of classified lines has now been extended to 340 lines of the V IV spectrum. Their wavelengths, visual intensities, wave numbers and classifications are given in table 3 . Only twenty of the observed lines still remain unclassified, and very probably they correspond to $4 f-5 g$ transitions.

Ionization Potential.-By using a Ritz formula $n^{*}=n+a+b T$ on the three observed members of the $3 d\left({ }^{2} \mathrm{D}\right) n s$ series an ionization potential of $376730 \pm 40$ $\mathrm{cm}^{-1}$ or 46.70 volts has been calculated. The levels and series used for the calculations of this limit are collected in table 4 . 
TABLE 1. Even levels of V IV

\begin{tabular}{|c|c|c|c|c|}
\hline Config. & Desig. & $J$ & Level & Interval \\
\hline $3 d^{2}$ & $3 d^{3} \mathrm{~F}$ & $\begin{array}{l}2 \\
3 \\
4\end{array}$ & $\begin{array}{r}0.0^{*} \\
325.4^{*} \\
734.7^{*}\end{array}$ & $\begin{array}{l}325.4 \\
409.3\end{array}$ \\
\hline $3 d^{2}$ & $3 d^{1} \mathrm{D}$ & 2 & $10959.3^{*}$ & \\
\hline $3 d^{2}$ & $3 d^{3} \mathrm{P}$ & $\begin{array}{l}0 \\
1 \\
2\end{array}$ & $\begin{array}{l}13122.8^{*} \\
13239.2^{*} \\
13458.3^{*}\end{array}$ & $\begin{array}{l}116.4 \\
219.1\end{array}$ \\
\hline $3 d^{2}$ & $3 d^{1} \mathrm{G}$ & 4 & $18391.2^{*}$ & \\
\hline $3 d^{2}$ & $3 d^{1} \mathrm{~S}$ & 0 & 42462.1 & \\
\hline $3 d\left({ }^{2} \mathrm{D}\right) 4 s$ & $4 s^{3} \mathrm{D}$ & $\begin{array}{l}1 \\
2 \\
3\end{array}$ & $\begin{array}{l}96196.1^{*} \\
96412.1^{*} \\
96798.0^{*}\end{array}$ & $\begin{array}{l}216.0 \\
385.7\end{array}$ \\
\hline $3 d\left({ }^{2} \mathrm{D}\right) 4 s$ & $4 s^{1} \mathrm{D}$ & 2 & $100200.7^{*}$ & \\
\hline $3 d\left({ }^{2} \mathrm{D}\right) 4 d$ & $4 d^{1} \mathrm{~F}$ & 3 & 215957.7 & \\
\hline $3 d\left({ }^{2} \mathrm{D}\right) 4 d$ & $4 d^{3} \mathrm{D}$ & $\begin{array}{l}1 \\
2 \\
3\end{array}$ & $\begin{array}{l}216905.0 \\
217108.0 \\
217350.0\end{array}$ & $\begin{array}{l}203.0 \\
242.0\end{array}$ \\
\hline $3 d\left({ }^{\circ} \mathrm{D}\right) 4 d$ & $4 d^{3} \mathrm{G}$ & $\begin{array}{l}3 \\
4 \\
5\end{array}$ & $\begin{array}{l}217836.3^{*} \\
218100.0^{*} \\
218463.6^{*}\end{array}$ & $\begin{array}{l}263.7 \\
363.6\end{array}$ \\
\hline $3 d\left({ }^{\circ} \mathrm{D}\right) 4 d$ & $4 d^{1} \mathrm{P}$ & 1 & 217990.7 & \\
\hline $3 d\left({ }^{2} \mathrm{D}\right) 4 d$ & $4 d^{3} \mathrm{~S}$ & 1 & 220343.5 & \\
\hline \multicolumn{5}{|c|}{297} \\
\hline
\end{tabular}


TABLE 1. Even levels of $\mathrm{V} \mathrm{IV}-$ Continued

\begin{tabular}{|c|c|c|c|c|}
\hline Config. & Desig. & $J$ & Level & Interval \\
\hline $3 d\left({ }^{2} \mathrm{D}\right) 4 d$ & $4 d^{3} \mathrm{~F}$ & $\begin{array}{l}2 \\
3 \\
4\end{array}$ & $\begin{array}{l}222794.6 \\
223033.0 \\
223304.6\end{array}$ & $\begin{array}{l}238.4 \\
271.6\end{array}$ \\
\hline $3 d\left({ }^{2} \mathrm{D}\right) 4 d$ & $4 d^{1} \mathrm{D}$ & 2 & 225804.1 & \\
\hline $3 d\left({ }^{2} \mathrm{D}\right) 4 d$ & $4 d^{3} \mathrm{P}$ & $\begin{array}{l}0 \\
1 \\
2\end{array}$ & $\begin{array}{l}226521.6 \\
226617.1 \\
226796.3\end{array}$ & $\begin{array}{r}95.5 \\
179.2\end{array}$ \\
\hline $3 d\left({ }^{2} \mathrm{D}\right) 4 d$ & $4 d^{1} \mathrm{G}$ & 4 & 227712.5 & \\
\hline $3 d\left({ }^{2} \mathrm{D}\right) 4 d$ & $4 d^{1} \mathrm{~S}$ & 0 & 234121.8 & \\
\hline $3 d\left({ }^{2} \mathrm{D}\right) 5 s$ & $5 s{ }^{3} \mathrm{D}$ & $\begin{array}{l}1 \\
2 \\
3\end{array}$ & $\begin{array}{l}236148.6 \\
236322.4 \\
236766.9\end{array}$ & $\begin{array}{l}173.8 \\
444.5\end{array}$ \\
\hline $3 d\left({ }^{2} \mathrm{D}\right) 5 s$ & $5 s^{1} \mathrm{D}$ & 2 & 237638.8 & \\
\hline $3 d\left({ }^{2} \mathrm{D}\right) 5 d$ & $5 d^{1} \mathrm{~F}$ & 3 & 283459.4 & \\
\hline $3 d\left({ }^{2} \mathrm{D}\right) 5 d$ & $5 d^{3} \mathrm{D}$ & $\begin{array}{l}1 \\
2 \\
3\end{array}$ & $\begin{array}{l}283722.7 \\
283940.4 \\
284226.7\end{array}$ & $\begin{array}{l}217.7 \\
286.3\end{array}$ \\
\hline $3 d\left({ }^{2} \mathrm{D}\right) 5 d$ & $5 d^{3} \mathrm{G}$ & $\begin{array}{l}3 \\
4 \\
5\end{array}$ & $\begin{array}{l}284101.1 \\
284340.1 \\
284699.3\end{array}$ & $\begin{array}{l}239.0 \\
359.2\end{array}$ \\
\hline $3 d\left({ }^{2} \mathrm{D}\right) 5 d$ & $5 d^{1} \mathrm{P}$ & 1 & 284365.7? & \\
\hline $3 d\left({ }^{2} \mathrm{D}\right) 5 d$ & $5 d^{3} \mathrm{~S}$ & 1 & 285298.6 & \\
\hline $3 d\left({ }^{2} \mathrm{D}\right) 5 d$ & $5 d^{3} \mathrm{~F}$ & $\begin{array}{l}2 \\
3 \\
4\end{array}$ & $\begin{array}{l}285798.9 \\
286056.9 \\
286286.5\end{array}$ & $\begin{array}{l}258.0 \\
229.6\end{array}$ \\
\hline
\end{tabular}


TABLE 1. Even levels of V IV-Continued

\begin{tabular}{|c|c|c|c|c|}
\hline Config. & Desig. & $J$ & Level & Interva \\
\hline $3 d\left({ }^{2} \mathrm{D}\right) 5 d$ & $5 d^{1} \mathrm{D}$ & 2 & 287221.4 & \\
\hline $3 d\left({ }^{2} \mathrm{D}\right) 5 d$ & $5 d^{3} \mathrm{P}$ & 2 & 287733.4 & \\
\hline $3 d\left({ }^{2} \mathrm{D}\right) 5 d$ & $5 d^{1} \mathrm{G}$ & 4 & 288127.6 & \\
\hline $3 d\left({ }^{2} \mathrm{D}\right) 6 s$ & $6 s^{3} \mathrm{D}$ & $\begin{array}{l}1 \\
2 \\
3\end{array}$ & $\begin{array}{l}291796.0 \\
291918.1 \\
292417.6\end{array}$ & $\begin{array}{l}122.1 \\
499.5\end{array}$ \\
\hline $3 d\left({ }^{2} \mathrm{D}\right) 6 s$ & $6 s^{1} \mathrm{D}$ & 2 & 292766.7 & \\
\hline $3 d\left({ }^{2} \mathrm{D}\right) 5 g$ & $5 g\left[3 \frac{1}{2}\right]$ & $\begin{array}{l}3 \\
4\end{array}$ & $\begin{array}{l}306323.1 \\
306327.7\end{array}$ & 4.6 \\
\hline $3 d\left({ }^{2} \mathrm{D}\right) 5 g$ & $5 g\left[4 \frac{1}{2}\right]$ & $\begin{array}{l}5 \\
4\end{array}$ & $\begin{array}{l}306871.0 \\
306876.3\end{array}$ & -5.3 \\
\hline
\end{tabular}

TABLE 2. Odd levels of V IV

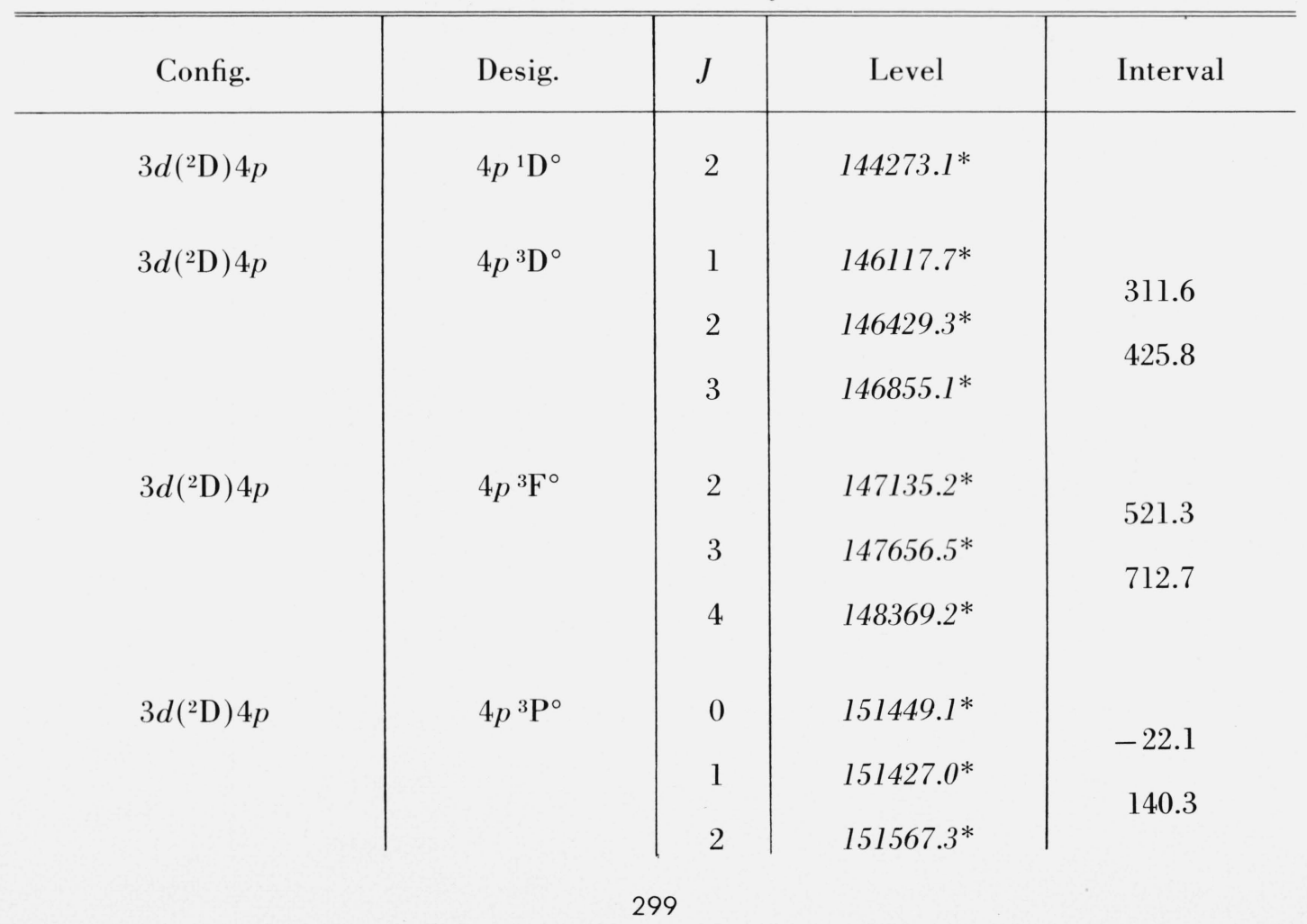


Table 2. Odd levels of V IV-Continued

\begin{tabular}{|c|c|c|c|c|}
\hline Config. & Desig. & $J$ & Level & Interval \\
\hline $3 d\left({ }^{2} \mathrm{D}\right) 4 p$ & $4 p^{1} \mathrm{~F}^{\circ}$ & 3 & $153918.7^{*}$ & \\
\hline $3 d\left({ }^{2} \mathrm{D}\right) 4 p$ & $4 p^{1} \mathrm{P}^{\circ}$ & 1 & $155565.5^{*}$ & \\
\hline $3 d\left({ }^{2} \mathrm{D}\right) 5 p$ & $5 p^{1} \mathrm{D}^{\circ}$ & 2 & 254468.8 & \\
\hline \multirow[t]{3}{*}{$3 d\left({ }^{2} \mathrm{D}\right) 5 p$} & $5 p^{3} \mathrm{D}^{\circ}$ & 1 & 254824.1 & \multirow{3}{*}{$\begin{array}{l}322.7 \\
298.7\end{array}$} \\
\hline & & 2 & 255146.8 & \\
\hline & & 3 & 255445.5 & \\
\hline \multirow[t]{3}{*}{$3 d\left({ }^{2} \mathrm{D}\right) 5 p$} & $5 p^{3} \mathrm{~F}^{\circ}$ & 2 & 255463.3 & \multirow{3}{*}{$\begin{array}{l}284.3 \\
504.1\end{array}$} \\
\hline & & 3 & 255747.6 & \\
\hline & & 4 & 256251.7 & \\
\hline \multirow[t]{3}{*}{$3 d\left({ }^{2} \mathrm{D}\right) 5 p$} & $5 p^{3} \mathrm{P}^{\circ}$ & 0 & 256739.9 & \multirow{3}{*}{$\begin{array}{r}41.9 \\
361.4\end{array}$} \\
\hline & & 1 & 256781.8 & \\
\hline & & 2 & 257143.2 & \\
\hline $3 d\left({ }^{2} \mathrm{D}\right) 5 p$ & $5 p{ }^{1} \mathrm{~F}^{\circ}$ & 3 & 257690.8 & \\
\hline $3 d\left({ }^{2} \mathrm{D}\right) 5 p$ & $5 p^{1} \mathrm{P}^{\circ}$ & 1 & 258288.8 & \\
\hline $3 d\left({ }^{2} \mathrm{D}\right) 4 f$ & $4 f^{1} \mathrm{G}^{\circ}$ & 4 & 263111.4 & \\
\hline \multirow[t]{3}{*}{$3 d\left({ }^{2} \mathrm{D}\right) 4 f$} & $4 f^{3} \mathrm{~F}^{\circ}$ & 2 & 263593.0 & \multirow{3}{*}{$\begin{array}{r}15.3 \\
504.8\end{array}$} \\
\hline & & 3 & 263608.3 & \\
\hline & & 4 & 264113.1 & \\
\hline \multirow[t]{3}{*}{$3 d\left({ }^{2} \mathrm{D}\right) 4 f$} & $4 f^{3} \mathrm{H}^{\circ}$ & 4 & 263822.4 & \multirow{3}{*}{$\begin{array}{r}339.4 \\
683.9\end{array}$} \\
\hline & & 5 & 264161.8 & \\
\hline & & 6 & 264845.7 & \\
\hline \multirow[t]{3}{*}{$3 d\left({ }^{2} \mathrm{D}\right) 4 f$} & $4 f^{3} \mathrm{G}^{\circ}$ & 3 & 263902.3 & \multirow{3}{*}{$\begin{array}{r}499.6 \\
190.0\end{array}$} \\
\hline & & 4 & 264401.9 & \\
\hline & & 5 & 264591.9 & \\
\hline
\end{tabular}


Table 2. Odd levels of V IV - Continued

\begin{tabular}{l|c|c|c|c}
\hline \hline Config. & Desig. & $J$ & Level & Interval \\
\hline $3 d\left({ }^{2} \mathrm{D}\right) 4 f$ & $4 f^{1} \mathrm{D}^{\circ}$ & 2 & 264482.8 & \\
$3 d\left({ }^{2} \mathrm{D}\right) 4 f$ & $4 f^{1} \mathrm{~F}^{\circ}$ & 3 & 264902.2 & \\
$3 d\left({ }^{2} \mathrm{D}\right) 4 f$ & $4 f^{3} \mathrm{D}^{\circ}$ & 1 & 265019.7 & 47.7 \\
& & 2 & 265067.4 & 204.2 \\
$3 d\left({ }^{2} \mathrm{D}\right) 4 f$ & $4 f^{3} \mathrm{P}^{\circ}$ & 1,2 & 265879.2 & \\
$3 d\left({ }^{2} \mathrm{D}\right) 4 f$ & $4 f^{1} \mathrm{H}^{\circ}$ & 5 & 266600.3 & \\
\hline
\end{tabular}

TABle 3. Classified lines of V IV

\begin{tabular}{|c|c|c|c|c|c|c|c|}
\hline$\lambda$ vac. & Intensity & Wave number & Classification & $\lambda$ vac. & Intensity & Wave number & Classification \\
\hline 675.469 & 30 & 148045.2 & $3 d^{3} \mathrm{~F}_{3}-4 p^{3} \mathrm{~F}_{4}^{\circ}$ & 722.912 & 40 & 138329.4 & $3 d^{3} \mathrm{P}_{1}-4 p^{3} \mathrm{P}_{2}^{\circ}$ \\
\hline 677.345 & 200 & 147635.2 & $3 d^{3} \mathrm{~F}_{4}-4 p^{3} \mathrm{~F}_{4}^{\circ}$ & 723.045 & 40 & 138303.9 & $3 d^{3} \mathrm{P}_{0}-4 p^{3} \mathrm{P}_{1}^{\circ}$ \\
\hline 678.740 & 60 & 147331.8 & $3 d^{3} \mathrm{~F}_{3}-4 p^{3} \mathrm{~F}_{3}^{\circ}$ & 723.537 & 40 & 138209.9 & $3 d^{3} \mathrm{P}_{1}-4 p^{3} \mathrm{P}_{0}^{\circ}$ \\
\hline 679.647 & 50 & 147135.2 & $3 d^{3} \mathrm{~F}_{2}-4 p^{3} \mathrm{~F}_{2}^{\circ}$ & 723.652 & 40 & 138188.0 & $3 d^{3} \mathrm{P}_{1}-4 p^{3} \mathrm{P}_{1}^{\circ}$ \\
\hline 680.632 & 40 & 146922.2 & $3 d^{3} \mathrm{~F}_{4}-4 p^{3} \mathrm{~F}_{3}^{\circ}$ & 724.068 & 40 & 138108.5 & $3 d^{3} \mathrm{P}_{2}-4 p^{3} \mathrm{P}_{2}^{\circ}$ \\
\hline 681.145 & 40 & 146811.6 & $3 d^{3} \mathrm{~F}_{3}-4 p^{3} \mathrm{~F}_{2}^{\circ}$ & 724.809 & 5 & 137967.3 & $3 d^{3} \mathrm{P}_{2}-4 p^{3} \mathrm{P}_{1}^{\circ}$ \\
\hline 682.455 & 40 & 146529.8 & $3 d^{3} \mathrm{~F}_{3}-4 p^{3} \mathrm{D}_{3}^{\circ}$ & 734.344 & 20 & 136175.9 & $3 d^{1} \mathrm{D}_{2}-4 p^{3} \mathrm{~F}_{2}^{\circ}$ \\
\hline 682.923 & 40 & 146429.3 & $3 d^{3} \mathrm{~F}_{2}-4 p^{3} \mathrm{D}_{2}^{\circ}$ & 737.854 & 400 & 135528.1 & $3 d^{1} \mathrm{G}_{4}-4 p^{1} \mathrm{~F}_{3}^{\circ}$ \\
\hline 684.368 & 500 & 146120.2 & $\left\{\begin{array}{l}3 d^{3} \mathrm{~F}_{4}-4 p^{3} \mathrm{D}_{3}^{\circ} \\
3 d^{3} \mathrm{~F}_{2}-4 p^{3} \mathrm{D}_{1}^{\circ}\end{array}\right.$ & $\begin{array}{l}745.165 \\
749.641\end{array}$ & $\begin{array}{l}20 \\
40\end{array}$ & $\begin{array}{l}134198.4 \\
133397.1\end{array}$ & $\begin{array}{l}3 d^{3} \mathrm{P}_{2}-4 p^{3} \mathrm{~F}_{3}^{\circ} \\
3 d^{3} \mathrm{P}_{2}-4 p^{3} \mathrm{D}_{3}^{\circ}\end{array}$ \\
\hline 684.450 & 100 & 146102.7 & $3 d^{3} \mathrm{~F}_{3}-4 p^{3} \mathrm{D}_{2}^{\circ}$ & 750.110 & 150 & 133313.7 & $3 d^{1} \mathrm{D}_{2}-4 p^{1} \mathrm{D}_{2}^{\circ}$ \\
\hline 691.530 & 100 & 144606.8 & $3 d^{1} \mathrm{D}_{1}-4 p^{1} \mathrm{P}_{1}^{\circ}$ & 750.809 & 40 & 133189.6 & $3 d^{3} \mathrm{P}_{1}-4 p^{3} \mathrm{D}_{2}^{\circ}$ \\
\hline 693.128 & 50 & 144273.4 & $3 d^{3} \mathrm{~F}_{2}-4 p^{1} \mathrm{D}_{2}^{\circ}$ & 751.908 & 30 & 132995.0 & $3 d^{3} \mathrm{P}_{0}-4 p^{3} \mathrm{D}_{1}^{\circ}$ \\
\hline 699.497 & 30 & 142959.8 & $3 d^{1} \mathrm{D}_{2}-4 p^{1} \mathrm{~F}_{3}^{\circ}$ & 752.038 & 30 & 132972.0 & $3 d^{3} \mathrm{P}_{2}-4 p^{3} \mathrm{D}_{2}^{\circ}$ \\
\hline 702.035 & 1 & 142443.0 & $3 d^{3} \mathrm{P}_{0}-4 p^{1} \mathrm{P}_{1}^{\circ}$ & 752.568 & 20 & 132878.3 & $3 d^{3} \mathrm{P}_{1}-4 p^{3} \mathrm{D}_{1}^{\circ}$ \\
\hline 711.911 & 20 & 140467.0 & $3 d^{1} \mathrm{D}_{2}-4 p^{3} \mathrm{P}_{1}^{\circ}$ & 778.433 & $\operatorname{tr}$ & 128463.2 & $3 d^{1} \mathrm{G}_{4}-4 p^{3} \mathrm{D}_{3}^{\circ}$ \\
\hline
\end{tabular}


TABLE 3. Classified lines of V IV-Continued

\begin{tabular}{|c|c|c|c|c|c|c|c|}
\hline$\lambda$ vac. & Intensity & Wave number & Classification & $\lambda$ vac. & Intensity & Wave number & Classification \\
\hline 884.146 & 30 & 113103.4 & $3 d^{1} \mathrm{~S}_{0}-4 p^{1} \mathrm{P}_{1}^{\circ}$ & 1326.807 & 5 & 75368.9 & $4 p^{3} \mathrm{P}_{1}^{\circ}-4 d^{3} \mathrm{P}_{2}$ \\
\hline 1071.054 & 20 & 93366.0 & $4 p^{1} \mathrm{D}_{2}^{\circ}-5 s{ }^{1} \mathrm{D}_{2}$ & 1329.288 & 10 & 75228.2 & $4 p^{3} \mathrm{P}_{2}^{\circ}-4 d^{3} \mathrm{P}_{2}$ \\
\hline 1086.382 & 5 & 92048.6 & $4 p^{1} \mathrm{D}_{2}^{\circ}-5 s^{3} \mathrm{D}_{2}$ & 1329.968 & 10 & 75189.8 & $4 p^{3} \mathrm{P}_{1}^{\circ}-4 d^{3} \mathrm{P}_{1}$ \\
\hline 1096.375 & 2 & 91209.7 & $4 p^{3} \mathrm{D}_{2}^{\circ}-5 s^{1} \mathrm{D}_{2}$ & 1330.355 & 10 & 75167.9 & $4 p^{3} \mathrm{P}_{0}^{\circ}-4 d^{3} \mathrm{P}_{1}$ \\
\hline 1110.720 & 2 & 90031.7 & $4 p^{3} \mathrm{D}_{1}^{\circ}-5 s^{3} \mathrm{D}_{1}$ & 1331.665 & $\operatorname{tr}$ & 75094.0 & $4 p^{3} \dot{\mathrm{P}_{1}^{\circ}}-4 d^{3} \mathrm{P}_{0}$ \\
\hline 1112.199 & 5 & 89912.0 & $4 p^{3} \mathrm{D}_{3}^{\circ}-5 s^{3} \mathrm{D}_{3}$ & 1332.459 & 3 & 75049.2 & $4 p^{3} \mathrm{P}_{2}^{\circ}-4 d^{3} \mathrm{P}_{1}$ \\
\hline 1112.436 & 5 & 89892.8 & $4 p^{3} \mathrm{D}_{2}^{\circ}-5 s^{3} \mathrm{D}_{2}$ & 1334.493 & ( ) & 74934.8 & $4 p^{3} \mathrm{~F}_{4}^{\circ}-4 d^{3} \mathrm{~F}_{4}$ \\
\hline 1127.836 & 20 & 88665.4 & $4 p^{3} \mathrm{~F}_{3}^{\circ}-5 s^{3} \mathrm{D}_{2}$ & 1339.335 & 5 & 74663.9 & $4 p^{3} \mathrm{~F}_{4}^{\circ}-4 d^{3} \mathrm{~F}_{3}$ \\
\hline 1131.255 & 20 & 88397.4 & $4 p^{3} \mathrm{~F}_{4}^{\circ}-5 s^{3} \mathrm{D}_{3}$ & 1344.493 & $\operatorname{tr}$ & 74377.5 & $4 p^{3} \mathrm{P}_{1}^{\circ}-4 d^{1} \mathrm{D}_{2}$ \\
\hline 1194.462 & 20 & 83719.7 & $4 p^{1} \mathrm{~F}_{3}^{\circ}-5 s^{1} \mathrm{D}_{2}$ & 1347.030 & 1 & 74237.4 & $4 p^{3} \mathrm{P}_{2}^{\circ}-4 d^{1} \mathrm{D}_{2}$ \\
\hline 1226.523 & 60 & 81531.3 & $4 p^{1} \mathrm{D}_{2}^{\circ}-4 d^{1} \mathrm{D}_{2}$ & 1355.131 & 80 & 73793.6 & $4 p^{1} \mathrm{~F}_{3}^{\circ}-4 d^{1} \mathrm{G}_{4}$ \\
\hline 1242.248 & 3 & 80499.2 & $4 p^{3} \mathrm{D}_{1}^{\circ}-4 d^{3} \mathrm{P}_{1}$ & 1356.529 & 10 & 73717.6 & $4 p^{1} \mathrm{D}_{2}^{\circ}-4 d^{1} \mathrm{P}_{1}$ \\
\hline 1243.718 & 10 & 80404.1 & $4 p^{3} \mathrm{D}_{1}^{\circ}-4 d^{3} \mathrm{P}_{0}$ & 1391.105 & 20 & 71885.3 & $4 p^{1} \mathrm{~F}_{3}^{\circ}-4 d^{1} \mathrm{D}_{2}$ \\
\hline 1244.287 & 2 & 80367.3 & $4 p^{3} \mathrm{D}_{2}^{\circ}-4 d^{3} \mathrm{P}_{2}$ & 1395.001 & 60 & 71684.5 & $4 p^{1} \mathrm{D}_{2}^{\circ}-4 d^{1} \mathrm{~F}_{3}$ \\
\hline 1247.069 & 30 & 80188.0 & $4 p^{3} \mathrm{D}_{2}^{\circ}-4 d^{3} \mathrm{P}_{1}$ & 1400.416 & 5 & 71407.3 & $4 p^{3} \mathrm{D}_{2}^{\circ}-4 d^{3} \mathrm{G}_{3}$ \\
\hline 1250.918 & 20 & 79941.3 & $4 p^{3} \mathrm{D}_{3}^{\circ}-4 d^{3} \mathrm{P}_{2}$ & 1403.618 & 8 & 71244.4 & $4 p^{3} \mathrm{D}_{3}^{\circ}-4 d^{3} \mathrm{G}_{4}$ \\
\hline 1271.153 & 2 & 78668.7 & $4 p^{3} \mathrm{~F}_{2}^{\circ}-4 d^{1} \mathrm{D}_{2}$ & 1408.639 & 8 & 70990.5 & $4 p^{3} \mathrm{D}_{1}^{\circ}-4 d^{3} \mathrm{D}_{2}$ \\
\hline 1272.972 & 30 & 78556.3 & $4 p^{1} \mathrm{P}_{1}^{\circ}-4 d^{1} \mathrm{~S}_{0}$ & 1410.018 & 8 & 70921.1 & $4 p^{3} \mathrm{D}_{3}^{\circ}-4 d^{3} \mathrm{D}_{2}$ \\
\hline 1273.529 & 10 & 78522.0 & $4 p^{1} \mathrm{D}_{2}^{\circ}-4 d^{3} \mathrm{~F}_{2}$ & 1412.686 & 20 & 70787.1 & $4 p^{3} \mathrm{D}_{1}^{\circ}-4 d^{3} \mathrm{D}_{1}$ \\
\hline 1304.173 & 30 & 76676.9 & $4 p^{3} \mathrm{D}_{1}^{\circ}-4 d^{3} \mathrm{~F}_{2}$ & 1414.409 & 50 & 70700.9 & $4 p^{3} \mathrm{~F}_{2}^{\circ}-4 d^{3} \mathrm{G}_{3}$ \\
\hline 1305.420 & 40 & 76603.7 & $4 p^{3} \mathrm{D}_{2}^{\circ}-4 d^{3} \mathrm{~F}_{3}$ & 1414.842 & 20 & 70679.3 & $4 p^{3} \mathrm{D}_{2}^{\circ}-4 d^{3} \mathrm{D}_{2}$ \\
\hline 1308.061 & 50 & 76449.0 & $4 p^{3} \mathrm{D}_{3}^{\circ}-4 d^{3} \mathrm{~F}_{4}$ & 1418.533 & 30 & 70495.4 & $4 p^{3} \mathrm{D}_{3}^{\circ}-4 d^{3} \mathrm{D}_{3}$ \\
\hline 1309.502 & 10 & 76364.9 & $4 p^{3} \mathrm{D}_{2}^{\circ}-4 d^{3} \mathrm{~F}_{2}$ & 1418.921 & 10 & 70476.1 & $4 p^{3} \mathrm{D}_{2}^{\circ}-4 d^{3} \mathrm{D}_{1}$ \\
\hline 1312.717 & 20 & 76177.9 & $4 p^{3} \mathrm{D}_{3}^{\circ}-4 d^{3} \mathrm{~F}_{3}$ & 1419.580 & 80 & 70443.4 & $4 p^{3} \mathrm{~F}_{3}^{\circ}-4 d^{3} \mathrm{G}_{4}$ \\
\hline 1317.566 & 5 & 75897.5 & $4 p^{3} \mathrm{~F}_{2}^{\circ}-4 d^{3} \mathrm{~F}_{3}$ & 1423.420 & 10 & 70253.3 & $4 p^{3} \mathrm{D}_{3}^{\circ}-4 d^{3} \mathrm{D}_{2}$ \\
\hline 1321.719 & 10 & 75659.0 & $4 p^{3} \mathrm{~F}_{2}^{\circ}-4 d^{3} \mathrm{~F}_{2}$ & 1423.719 & 30 & 70238.6 & $4 p^{1} \mathrm{P}_{1}^{\circ}-4 d^{1} \mathrm{D}_{2}$ \\
\hline 1321.917 & 10 & 75647.7 & $4 p^{3} \mathrm{~F}_{3}^{\circ}-4 d^{3} \mathrm{~F}_{4}$ & 1424.197 & $\operatorname{tr}$ & 70215.0 & $4 p^{3} \mathrm{~F}_{2}^{\circ}-4 d^{3} \mathrm{D}_{3}$ \\
\hline 1326.666 & 5 & 75376.9 & $4 p^{3} \mathrm{~F}_{3}^{\circ}-4 d^{3} \mathrm{~F}_{3}$ & 1424.916 & 10 & 70179.4 & $4 p^{3} \mathrm{~F}_{3}^{\circ}-4 d^{3} \mathrm{G}_{3}$ \\
\hline
\end{tabular}


TABLE 3. Classified lines of V IV-Continued

\begin{tabular}{|c|c|c|c|c|c|c|c|}
\hline$\lambda$ vac. & Intensity & W ave number & Classification & $\lambda$ vac. & Intensity & Wave number & Classification \\
\hline 1426.654 & 100 & 70094.1 & $4 p^{3} \mathrm{~F}_{4}^{\circ}-4 d^{3} \mathrm{G}_{5}$ & 1963.103 & 300 & 50939.8 & $4 s^{3} \mathrm{D}_{1}-4 p^{3} \mathrm{~F}_{2}^{\circ}$ \\
\hline 1429.114 & 10 & 69973.4 & $4 p^{3} \mathrm{~F}_{2}^{\circ}-4 d^{3} \mathrm{D}_{2}$ & 1966.244 & 20 & 50858.4 & $4 s^{3} \mathrm{D}_{3}-4 p^{3} \mathrm{~F}_{3}^{\circ}$ \\
\hline 1433.276 & 1 & 69770.2 & $4 p^{3} \mathrm{~F}_{2}^{\circ}-4 d^{3} \mathrm{D}_{1}$ & 1971.471 & 40 & 50723.5 & $4 s^{3} \mathrm{D}_{2}-4 p^{3} \mathrm{~F}_{2}^{\circ}$ \\
\hline 1434.092 & 15 & 69730.5 & $4 p^{3} \mathrm{~F}_{4}^{\circ}-4 d^{3} \mathrm{G}_{4}$ & 1982.422 & 15 & 50443.3 & $4 s^{3} \mathrm{D}_{2}-4 p^{3} \mathrm{D}_{3}^{\circ}$ \\
\hline 1434.842 & 15 & 69694.1 & $4 p^{3} \mathrm{~F}_{3}^{\circ}-4 d^{3} \mathrm{D}_{3}$ & 1990.712 & 40 & 50233.3 & $4 s^{3} \mathrm{D}_{1}-4 p^{3} \mathrm{D}_{2}^{\circ}$ \\
\hline 1439.834 & 1 & 69452.5 & $4 p^{3} \mathrm{~F}_{3}^{\circ}-4 d^{3} \mathrm{D}_{2}$ & 1997.722 & 500 & 50057.0 & $4 s^{3} \mathrm{D}_{3}-4 p^{3} \mathrm{D}_{3}^{\circ}$ \\
\hline 1447.120 & $\operatorname{tr}$ & 69102.8 & $4 p^{3} \mathrm{D}_{3}^{\circ}-4 d^{1} \mathrm{~F}_{3}$ & 1999.320 & 200 & 50017.0 & $4 s^{3} \mathrm{D}_{2}-4 p^{3} \mathrm{D}_{2}^{\circ}$ \\
\hline 1449.681 & 20 & 68980.7 & $4 p^{3} \mathrm{~F}_{4}^{\circ}-4 d^{3} \mathrm{D}_{3}$ & $\lambda$ air & & & \\
\hline 1451.042 & 30 & 68916.0 & $4 p^{3} \mathrm{P}_{1}^{\circ}-4 d^{3} \mathrm{~S}_{1}$ & 2002.480 & 100 & 49921.9 & $4 s^{3} \mathrm{D}_{1}-4 p^{3} \mathrm{D}_{1}^{\circ}$ \\
\hline 1451.496 & 10 & 68894.5 & $4 p^{3} \mathrm{P}_{0}^{\circ}-4 d^{3} \mathrm{~S}_{1}$ & 2011.180 & 40 & 49706.0 & $4 s^{3} \mathrm{D}_{2}-4 p^{3} \mathrm{D}_{1}^{\circ}$ \\
\hline 1454.000 & 40 & 68775.8 & $4 p^{3} \mathrm{P}_{2}^{\circ}-4 d^{3} \mathrm{~S}_{1}$ & 2014.199 & 40 & 49631.5 & $4 s^{3} \mathrm{D}_{3}-4 p^{3} \mathrm{D}_{2}^{\circ}$ \\
\hline 1520.142 & 60 & 65783.3 & $4 p^{3} \mathrm{P}_{2}^{\circ}-4 d^{3} \mathrm{D}_{3}$ & 2027.144 & 1 & 49314.6 & $4 d^{1} \mathrm{~F}_{3}-4 f^{3} \mathrm{D}_{3}^{\circ}$ \\
\hline 1522.493 & 40 & 65681.7 & $4 p^{3} \mathrm{P}_{1}^{\circ}-4 d^{3} \mathrm{D}_{2}$ & 2042.454 & $20 H$ & 48945.0 & $4 d^{1} \mathrm{~F}_{3}-4 f^{1} \mathrm{~F}_{3}^{\circ}$ \\
\hline 1525.756 & 10 & 65541.3 & $4 p^{3} \mathrm{P}_{2}^{\circ}-4 d^{3} \mathrm{D}_{2}$ & 2060.113 & $\operatorname{tr} H$ & 48525.5 & $4 d^{1} \mathrm{~F}_{3}-4 f^{1} \mathrm{D}_{2}^{\circ}$ \\
\hline 1527.223 & 15 & 65478.3 & $4 p^{3} \mathrm{P}_{1}^{\circ}-4 d^{3} \mathrm{D}_{1}$ & 2063.563 & $2 H$ & 48444.4 & $4 d^{1} \mathrm{~F}_{3}-4 f^{3} \mathrm{G}_{4}^{\circ}$ \\
\hline 1527.721 & 15 & 65457.0 & $4 p^{3} \mathrm{P}_{0}^{\circ}-4 d^{3} \mathrm{D}_{1}$ & 2079.300 & 30 & 48077.8 & $4 s^{3} \mathrm{D}_{1}-4 p^{1} \mathrm{D}_{2}^{\circ}$ \\
\hline 1601.915 & 80 & 62425.3 & $4 p^{1} \mathrm{P}_{1}^{\circ}-4 d^{1} \mathrm{P}_{1}$ & 2084.433 & $20 H$ & 47959.4 & $4 d^{3} \mathrm{D}_{2}-4 f^{3} \mathrm{D}_{2}^{\circ}$ \\
\hline 1611.879 & 80 & 62039.4 & $4 p^{1} \mathrm{~F}_{3}^{\circ}-4 d^{1} \mathrm{~F}_{3}$ & 2086.073 & $30 H$ & 47921.7 & $4 d^{3} \mathrm{D}_{3}-4 f^{3} \mathrm{D}_{3}^{\circ}$ \\
\hline 1806.184 & 80 & 55365.3 & $4 s^{1} \mathrm{D}_{1}-4 p^{1} \mathrm{P}_{1}^{\circ}$ & 2088.737 & $50 h$ & 47860.6 & $4 s^{3} \mathrm{D}_{2}-4 p^{1} \mathrm{D}_{2}^{\circ}$ \\
\hline 1809.854 & 60 & 55253.1 & $4 s^{3} \mathrm{D}_{1}-4 p^{3} \mathrm{P}_{0}^{\circ}$ & 2105.709 & $\operatorname{tr}$ & 47474.9 & $4 s^{3} \mathrm{D}_{3}-4 p^{1} \mathrm{D}_{2}^{\circ}$ \\
\hline 1810.566 & 30 & 55231.3 & $4 s^{3} \mathrm{D}_{1}-4 p^{3} \mathrm{P}_{1}^{\circ}$ & 2106.560 & 2 & 47455.7 & $4 s^{1} \mathrm{D}_{2}-4 p^{3} \mathrm{~F}_{3}^{\circ}$ \\
\hline 1813.050 & 50 & 55155.7 & $4 s{ }^{3} \mathrm{D}_{2}-4 p^{3} \mathrm{P}_{2}^{\circ}$ & 2120.052 & $40 H$ & 47153.7 & $4 d^{1} \mathrm{~F}_{3}-4 f^{1} \mathrm{G}_{4}^{\circ}$ \\
\hline 1817.676 & 100 & 55015.3 & $4 s^{3} \mathrm{D}_{2}-4 p^{3} \mathrm{P}_{1}^{\circ}$ & 2129.934 & 30 & 46935.0 & $4 s^{1} \mathrm{D}_{2}-4 p^{3} \mathrm{~F}_{2}^{\circ}$ \\
\hline 1825.836 & 200 & 54769.4 & $4 s^{3} \mathrm{D}_{3}-4 p^{3} \mathrm{P}_{2}^{\circ}$ & 2136.330 & $10 h$ & 46794.5 & $4 d^{3} \mathrm{D}_{2}-4 f^{3} \mathrm{G}_{3}^{\circ}$ \\
\hline 1861.558 & 300 & 53718.4 & $4 s^{1} \mathrm{D}_{2}-4 p^{1} \mathrm{~F}_{3}^{\circ}$ & 2137.741 & $20 h$ & 46763.6 & $4 d^{3} \mathrm{D}_{3}-4 f^{3} \mathbf{F}_{4}^{\circ}$ \\
\hline 1939.065 & 500 & 51571.2 & $4 s^{3} \mathrm{D}_{3}-4 p^{3} \mathrm{~F}_{4}^{\circ}$ & 2141.199 & $40 H$ & 46688.1 & $4 d^{3} \mathrm{D}_{1}-4 f^{3} \mathrm{~F}_{2}^{\circ}$ \\
\hline 1946.772 & 5 & 51367.1 & $4 s^{1} \mathrm{D}_{2}-4 p^{3} \mathrm{P}_{2}^{\circ}$ & 2146.828 & $50 \mathrm{H}$ & 46565.7 & $4 d^{3} \mathrm{G}_{3}-4 f^{3} \mathrm{G}_{4}^{\circ}$ \\
\hline 1951.432 & 400 & 51244.4 & $4 s^{3} \mathbf{D}_{2}-4 p^{3} \mathbf{F}_{3}^{\circ}$ & 2149.852 & $20 H$ & 46500.2 & $4 d^{3} \mathrm{D}_{2}-4 f^{3} \mathrm{~F}_{3}^{\circ}$ \\
\hline
\end{tabular}


TABLE 3. Classified lines of V IV-Continued

\begin{tabular}{|c|c|c|c|c|c|c|c|}
\hline$\lambda$ air & Intensity & Wave number & Classification & $\lambda$ air & Intensity & Wave number & Classification \\
\hline 2150.231 & $40 \mathrm{H}$ & 46492.0 & $\left\{\begin{array}{l}4 d^{3} \mathrm{G}_{4}-4 f^{3} \mathrm{G}_{5}^{\circ} \\
4 d^{1} \mathrm{P}-4 f^{1} \mathrm{D}^{\circ}\end{array}\right.$ & 2384.729 & $10 H$ & 41920.7 & $4 f^{3} G_{4}^{\circ}-5 g\left[3^{\frac{1}{2}}\right]_{3}$ \\
\hline & & & $\left(4 d^{1} \mathrm{P}_{1}-4 f^{1} \mathrm{D}_{2}^{2}\right.$ & 2387.663 & $3 H$ & 41869.2 & $4 d^{3} \mathrm{~F}_{3}-4 f^{1} \mathrm{~F}_{3}^{\circ}$ \\
\hline 2151.087 & $20 h$ & 46473.5 & $4 d^{3} \mathrm{D}_{3}-4 \cdot f^{3} \mathrm{H}_{4}^{\circ}$ & 2395.450 & $10 H$ & 41733.1 & $4 d^{1} \mathrm{~F}_{3}-5 p^{1} \mathbf{F}_{3}^{\circ}$ \\
\hline 2155.336 & $100 \mathrm{H}$ & 46381.9 & $4 d^{3} \mathrm{G}_{5}-4 f^{3} \mathrm{H}_{6}^{\circ}$ & & $5 H$ & 41604.5 & $4 f^{3} \mathrm{D}_{3}^{\circ}-5 g\left[4 \frac{1}{2}\right]$ \\
\hline 2159.055 & $10 H$ & 46302.0 & $4 d^{3} \mathrm{G}_{4}-4 f^{3} \mathrm{G}_{4}^{\circ}$ & 2413.256 & $20 H$ & 41425.2 & $4 f^{3} \mathrm{G}_{3}^{\circ}-5 g\left[3 \frac{1}{2}\right]$ \\
\hline 2160.222 & $20 H$ & 46277.0 & $4 d^{3} \mathrm{G}_{3}-4 f^{3} \mathbf{F}_{4}^{\circ}$ & & $5 H$ & 41420.6 & $4 f^{1} \mathrm{~F}_{3}^{\circ}-5 g\left[3 \frac{1}{2}\right]:$ \\
\hline 2162.498 & 30 & 46228.3 & $4 s^{1} \mathrm{D}_{2}-4 p^{3} \mathrm{D}_{2}^{\circ}$ & 2416.552 & $30 h$ & 41368.7 & $4 d^{3} \mathrm{~F}_{3}-4 f^{3} \mathrm{G}_{4}^{\circ}$ \\
\hline 2167.200 & $20 H$ & 46128.0 & $4 d^{3} \mathrm{G}_{5}-4 f^{3} \mathrm{G}_{5}^{\circ}$ & 27.0 .002 & $50 h$ & 41281.3 & $4 d^{3} \mathrm{~F}_{4}-4 f^{3} \mathrm{G}_{5}^{\circ}$ \\
\hline 2170.384 & $(40 H)$ & 46060.4 & $4 d^{3} \mathrm{G}_{4}-4 f^{3} \mathrm{H}_{5}^{\circ}$ & & 50 & F11201.0 & \\
\hline 2173.893 & $10 \mathrm{H}$ & 45986.0 & $4 d^{3} \mathrm{G}_{3}-4 f^{3} \mathrm{H}_{4}^{\circ}$ & 243 & 30 & 10 & \\
\hline 2186.394 & $\operatorname{tr} H$ & 45723.1 & $4 d^{3} \mathrm{G}_{4}-4 f^{3} \mathrm{H}_{4}^{\circ}$ & 243 & $10 h$ & 41097.2 & $4 d^{3} \mathrm{~F}_{4}-4 f^{3} \mathrm{G}_{4}^{\circ}$ \\
\hline 2187.562 & $5 H$ & 45698.7 & $4 d^{3} \mathrm{G}_{5}-4 f^{3} \mathrm{H}_{5}^{\circ}$ & 2433.530 & $50 h$ & 41080.1 & $4 d^{3} \mathrm{~F}_{3}-4 f^{3} \mathbf{F}_{4}^{\circ}$ \\
\hline 2195.388 & $10 H$ & 45535.8 & $4 d^{3} \mathrm{~S}_{1}-4 f^{3} \mathrm{P}_{2}^{\circ}$ & 244 & 30 & 40809.5 & $4 d^{3} \mathrm{~F}_{3}-4 f^{3} \mathrm{C}$ \\
\hline 2268.298 & 500 & 44072.3 & & 2446.802 & $50 h$ & 40857.3 & $4 d^{3} \mathrm{~F}_{4}-4 f^{3} \mathrm{H}_{5}^{\circ}$ \\
\hline 2313.236 & $1 H$ & 43216.2 & $4 f^{1} \mathrm{G}_{4}^{\circ}-5 g\left[3^{\frac{1}{2}}\right]_{4}$ & 2449.404 & $40 h$ & 40813.9 & $4 d^{3} \mathrm{~F}_{2}-4$ \\
\hline 2321.962 & $1 H$ & 43053.8 & $4 f^{3} \mathrm{H}_{4}^{\circ}-5 g\left[4 \frac{1}{2}\right]_{4}$ & 2449.723 & $20 h$ & 4( & $4 d^{3} \mathrm{~F}_{4}-4 f^{3} \mathrm{~F}_{4}^{\circ}$ \\
\hline 2322.259 & $5 H$ & 43048.3 & $4 f^{3} \mathrm{H}_{4}^{\circ}-5 g\left[4 \frac{1}{2}\right]_{5}$ & 245 & 201 & 406 & $4 d^{3} \mathrm{~F}_{2}-4$ \\
\hline 2326.291 & $3 H$ & 42973.7 & $4 f^{3} \mathrm{G}_{3}^{\circ}-5 g\left[4 \frac{1}{2}\right]_{4}$ & $\begin{array}{l}2450.869 \\
2463.796\end{array}$ & $10 h$ & 40575.5 & $4 d^{3} \mathrm{~F}_{3}-4 f^{3} \mathrm{~F}_{3}^{\circ}$ \\
\hline 2338.032 & $10 H$ & 42757.9 & $4 f^{3} \mathrm{~F}_{4}^{\circ}-5 g\left[4 \frac{1}{2}\right]_{5}$ & 2464.720 & $2 h$ & 40560.3 & $4 d^{3} \mathrm{~F}_{3}-4 f^{3} \mathrm{~F}_{2}^{\circ}$ \\
\hline 2339.548 & $20 H$ & 42730.2 & $4 f^{3} \mathrm{~F}_{2}^{\circ}-5 g\left[3 \frac{1}{2}\right]_{3}$ & 2467287 & $20 h$ & 405181 & 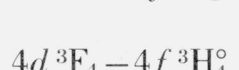 \\
\hline 2340.140 & $10 H$ & 42719.4 & $4 f^{3} \mathbf{F}_{3}^{\circ}-5 g\left[3 \frac{1}{2}\right]_{4}$ & & & & \\
\hline 2340.704 & $5 H$ & 42709.1 & $4 f^{3} \mathrm{H}_{5}^{\circ}-5 g\left[4 \frac{1}{2}\right]_{5}$ & 19 & $1 H$ & 40341.0 & $4 d^{3} \mathrm{D}_{3}-5 p^{1} \mathrm{~F}_{3}^{\circ}$ \\
\hline 2351.934 & $5 H$ & 42505.2 & $4 f^{3} \mathrm{H}_{4}^{\circ}-5 g\left[3 \frac{1}{2}\right]_{4}$ & 2480.739 & $30 h$ & 40298.4 & $4 d^{1} \mathrm{P}_{1}-5 p^{1} \mathrm{P}_{1}^{\circ}$ \\
\hline 2353.639 & $3 H$ & 42474.4 & $4 f^{3} \mathrm{G}_{4}^{\circ}-5 g\left[4 \frac{1}{2}\right]_{4}$ & 2494.351 & $20 h$ & 40078.5 & $4 d^{3} \mathrm{~F}_{3}-4 f^{1} \mathrm{G}_{4}^{\circ}$ \\
\hline 2356.369 & $10 H$ & 42425.2 & $4 f^{3} \mathrm{G}_{3}^{\circ}-5 g\left[3 \frac{1}{2}\right]_{4}$ & 2497.049 & $10 h$ & 40035.2 & $4 d^{3} \mathrm{D}_{2}-5 p^{3} \mathrm{P}_{2}^{\circ}$ \\
\hline 2356.624 & $5 H$ & 42420.6 & $4 f^{3} \mathrm{G}_{3}^{\circ}-5 g\left[3^{\frac{1}{2}}\right]_{3}$ & 2506.969 & $10 h$ & 39876.8 & $4 d^{3} \mathrm{D}_{1}-5 p^{3} \mathrm{P}_{1}^{\circ}$ \\
\hline 2364.512 & $1 H$ & 42279.1 & $4 f^{3} \mathrm{G}_{5}^{\circ}-5 g\left[4 \frac{1}{2}\right]$. & 2509.606 & $5 h$ & 39834.9 & $4 d^{3} \mathrm{D}_{1}-5 p^{3} \mathrm{P}_{0}^{\circ}$ \\
\hline 2378.290 & $1 H$ & 42034.2 & $4 d^{3} \mathrm{~F}_{3}-4 f^{3} \mathrm{D}_{2}^{\circ}$ & 2511.377 & $1 H$ & 39806.8 & $4 d^{3} \mathrm{~F}_{4}-4 f^{1} \mathrm{G}_{4}^{\circ}$ \\
\hline 2381.712 & $10 H$ & 41973.8 & $4 f^{1} \mathrm{~F}_{3}^{\circ}-5 g\left[4 \frac{1}{2}\right]_{4}$ & 2512.242 & ( ) & 39793.1 & $4 d^{3} \mathrm{D}_{3}-5 p^{3} \mathrm{P}_{2}^{\circ}$ \\
\hline
\end{tabular}


TABLE 3. Classified lines of V IV-Continued

\begin{tabular}{|c|c|c|c|c|c|c|c|}
\hline$\lambda$ air & Intensity & Wave number & Classification & $\lambda$ air & Intensity & $\mathrm{W}$ ave number & Classification \\
\hline 2519.803 & $20 h$ & 39673.7 & $4 d^{3} \mathrm{D}_{2}-5 p^{3} \mathrm{P}_{1}^{\circ}$ & 2650.613 & $8 h$ & 37715.9 & $4 d^{3} \mathrm{D}_{2}-5 p^{3} \mathrm{D}_{1}^{\circ}$ \\
\hline 2530.520 & $2 h$ & 39505.7 & $4 d^{1} \mathrm{~F}_{3}-5 p^{3} \mathrm{~F}_{2}^{\circ}$ & 2655.408 & $50 \mathrm{H}$ & 37647.8 & $4 d^{3} \mathrm{G}_{4}-5 p^{3} \mathrm{~F}_{3}^{\circ}$ \\
\hline 2532.982 & $20 h$ & 39467.3 & $4 d^{1} \mathrm{D}_{2}-4 f^{3} \mathrm{D}_{3}^{\circ}$ & 2656.868 & $50 \mathrm{H}$ & 37627.1 & $4 d^{3} \mathrm{G}_{3}-5 p^{3} \mathrm{~F}_{2}^{\circ}$ \\
\hline 2546.228 & $20 h$ & 39262.0 & $4 d^{3} \mathrm{P}_{1}-4 f^{3} \mathrm{P}_{2}^{\circ}$ & 2667.837 & $1 h$ & 37472.4 & $4 d^{1} \mathrm{P}_{1}-5 p^{3} \mathrm{~F}_{2}^{\circ}$ \\
\hline 2550.971 & $2 H$ & 39189.0 & $4 d^{1} \mathrm{~F}_{3}-5 p^{3} \mathrm{D}_{2}^{\circ}$ & 2669.483 & $10 h$ & 37449.3 & $5 p^{1} \mathrm{D}_{2}^{\circ}-6 s^{3} \mathrm{D}_{2}$ \\
\hline 2556.915 & $50 h$ & 39097.9 & $4 d^{1} \mathrm{D}_{2}-4 f^{1} \mathrm{~F}_{3}^{\circ}$ & 2703.933 & $20 \mathrm{H}$ & 36972.2 & $\left\{5 p^{3} \mathrm{D}_{3}^{\circ}-6 s^{3} \mathrm{D}_{3}\right.$ \\
\hline 2557.897 & $15 h$ & 39082.9 & $4 d^{3} \mathrm{P}_{2}-4 f^{3} \mathrm{P}_{2}^{\circ}$ & & & & $5 p$ \\
\hline 2569.812 & $10 h$ & 38901.7 & $4 d^{3} \mathrm{D}_{3}-5 p^{3} \mathrm{~F}_{4}^{\circ}$ & 2716.594 & $20 h$ & 36799.9 & $4 d^{3} \mathrm{~S}_{1}-5 p^{3} \mathrm{P}_{2}^{\circ}$ \\
\hline 2570.724 & $80 h$ & 38887.9 & $4 d^{1} \mathrm{G}_{4}-4 f^{1} \mathrm{H}_{5}^{\circ}$ & 2718.722 & $2 h$ & 36771.1 & $5 p^{3} \mathrm{D}_{2}^{\circ}-6 s^{3} \mathrm{D}_{2}$ \\
\hline 2584.636 & $40 h$ & 38678.6 & $4 d^{1} \mathrm{D}_{2}-4 f^{1} \mathrm{D}_{2}^{\circ}$ & 2727.780 & $1 h$ & 36649.0 & $5 p^{3} \mathrm{D}_{2}^{\circ}-6 s^{3} \mathrm{D}_{1}$ \\
\hline 2587.258 & $10 h$ & 38639.4 & $4 d^{3} \mathrm{D}_{2}-5 p^{3} \mathrm{~F}_{3}^{\circ}$ & 2740.545 & $5 h$ & 36478.3 & $4 d^{1} \mathrm{P}_{1}-5 p^{1} \mathrm{D}_{2}^{\circ}$ \\
\hline 2592.747 & ( ) & 38557.6 & $4 d^{3} \mathrm{D}_{1}-5 p^{3} \mathrm{~F}_{2}^{\circ}$ & 2740.966 & $5 h$ & 36472.7 & $5 p^{3} \mathrm{D}_{3}^{\circ}-6 s^{3} \mathrm{D}_{2}$ \\
\hline 2595.858 & $20 H$ & 38511.4 & $4 d^{1} \mathrm{~F}_{3}-5 p^{1} \mathrm{D}_{2}^{\circ}$ & 2743.523 & $20 h$ & 36438.7 & $4 d^{3} \mathrm{~S}_{1}-5 p^{3} \mathrm{P}_{1}^{\circ}$ \\
\hline 2596.761 & $15 h$ & 38498.0 & $4 d^{3} \mathrm{P}_{0}-4 f^{3} \mathrm{D}_{1}^{\circ}$ & 2751.528 & $10 h$ & 36332.7 & $5 p^{3} \mathrm{~F}_{2}^{\circ}-6 s^{3} \mathrm{D}_{1}$ \\
\hline 2598.287 & $30 h$ & 38475.4 & $4 d^{3} \mathrm{P}_{2}-4 f^{3} \mathrm{D}_{3}^{\circ}$ & 2763.860 & $15 h$ & 36170.6 & $5 p^{3} \mathrm{~F}_{3}^{\circ}-6 s^{3} \mathrm{D}_{2}$ \\
\hline 2599.983 & $30 h$ & 38450.3 & $4 d^{3} \mathrm{P}_{1}-4 f^{3} \mathrm{D}_{2}^{\circ}$ & 2764.219 & $15 h$ & 36165.9 & $5 p^{3} \mathrm{~F}_{4}^{\circ}-6 s^{3} \mathrm{D}_{3}$ \\
\hline 2603.213 & $10 h$ & 38402.6 & $4 d^{3} \mathrm{P}_{1}-4 f^{3} \mathrm{D}_{1}^{\circ}$ & 282 & $20 h$ & 35398.7 & $4 d^{1} \mathrm{G}_{4}-4$ \\
\hline 2607.633 & $5 h$ & 38337.5 & $4 d^{3} \mathrm{D}_{2}-5 p^{3} \mathrm{D}_{3}^{\circ}$ & 2834.089 & $5 H$ & 35274.3 & $5 p^{3} \mathrm{P}_{2}^{0}-6 s$ \\
\hline 2610.323 & $10 h$ & 38298.0 & $5 p^{1} \mathrm{D}_{2}^{\circ}-6 s^{1} \mathrm{D}_{2}$ & $\begin{array}{l}2850.160 \\
2899.575\end{array}$ & $\begin{array}{l}2 H \\
2 h\end{array}$ & $\begin{array}{l}35075.4 \\
34477.7\end{array}$ & $\begin{array}{l}5 p^{1} \mathrm{~F}_{3}^{\circ}-6 s^{1} \mathrm{D}_{2} \\
5 p^{1} \mathrm{P}_{1}^{\circ}-6 s^{1} \mathrm{D}_{2}\end{array}$ \\
\hline 2614.159 & $1 h$ & 38241.8 & $4 d^{3} \mathrm{D}_{1}-5 p^{3} \mathrm{D}_{2}^{\circ}$ & 3034.27 & 10 & 32947.3 & $4 d^{3} \mathrm{~F}_{4}-5 p^{3} \mathrm{~F}_{4}^{\circ}$ \\
\hline 2620.320 & $25 h$ & 38151.9 & $4 d^{3} \mathrm{G}_{4}-5 p^{3} \mathrm{~F}_{4}^{\circ}$ & 3052.346 & 10 & 32752.16 & $5 p^{1} \mathrm{D}_{2}^{\circ}-5 d^{1} \mathrm{D}_{2}$ \\
\hline 2623.483 & $15 h$ & 38105.9 & $4 d^{3} \mathrm{P}_{2}-4 f^{1} \mathrm{~F}_{3}^{\circ}$ & 3055.864 & 5 & 32714.46 & $4 d^{3} \mathrm{~F}_{3}-5 p^{3} \mathrm{~F}_{3}^{\circ}$ \\
\hline 2624.213 & $50 h$ & 38095.3 & $4 d^{3} \mathrm{D}_{3}-5 p^{3} \mathrm{D}_{3}^{\circ}$ & 3060.146 & 5 & 32668.68 & $4 d^{3} \mathrm{~F}_{2}-5 p^{3} \mathrm{~F}_{2}^{\circ}$ \\
\hline 2628.090 & $20 h$ & 38039.1 & $4 d^{3} \mathrm{D}_{2}-5 p^{3} \mathrm{D}_{2}^{\circ}$ & 3067.85 & $\operatorname{tr} ?$ & 32586.6 & $5 p^{3} \mathrm{D}_{2}^{\circ}-5 d^{3} \mathrm{P}_{2}$ \\
\hline 2636.401 & $30 h$ & 37919.2 & $4 d^{3} \mathrm{D}_{1}-5 p^{3} \mathrm{D}_{1}^{\circ}$ & 3077.476 & 15 & 32484.72 & $4 d^{1} \mathrm{D}_{2}-5 p^{1} \mathrm{P}_{1}^{\circ}$ \\
\hline 2636.936 & $10 h$ & 37911.5 & $4 d^{3} \mathrm{G}_{3}-5 p^{3} \mathrm{~F}_{3}^{\circ}$ & 3084.36 & 1 & 32412.2 & $4 d^{3} \mathrm{~F}_{3}-5 p^{3} \mathrm{D}_{3}^{\circ}$ \\
\hline 2644.946 & $8 h$ & 37796.7 & $4 d^{3} \mathrm{D}_{3}-5 p^{3} \mathrm{D}_{2}^{\circ}$ & 3096.226 & 2 & 32288.01 & $5 p^{3} \mathrm{D}_{3}^{\circ}-5 d^{3} \mathrm{P}_{2}$ \\
\hline 2645.541 & $80 H$ & 37788.2 & $4 d^{3} \mathrm{G}_{5}-5 p^{3} \mathrm{~F}_{4}^{\circ}$ & 3110.416 & 30 & 32140.72 & $4 d^{3} \mathbf{F}_{4}-5 p^{3} \mathrm{D}_{3}^{\circ}$ \\
\hline
\end{tabular}


TABLE 3. Classified lines of V IV-Continued

\begin{tabular}{|c|c|c|c|c|c|c|c|}
\hline$\lambda$ air & Intensity & Wave number & Classification & $\lambda$ air & Intensity & Wave number & Classification \\
\hline 3113.022 & 25 & 32113.81 & $4 d^{3} \mathrm{~F}_{3}-5 p^{3} \mathrm{D}_{2}^{\circ}$ & 3489.51 & $\operatorname{tr}$ & 28649.1 & $4 d^{3} \mathrm{P}_{2}-5 p^{3} \mathrm{D}_{3}^{\circ}$ \\
\hline 3121.304 & 10 & 32028.60 & $4 d^{3} \mathrm{~F}_{2}-5 p^{3} \mathrm{D}_{1}^{\circ}$ & 3490.913 & 30 & 28637.61 & $5 p^{3} \mathrm{~F}_{2}^{\circ}-5 d^{3} \mathrm{G}_{3}$ \\
\hline 3135.192 & 20 & 31886.73 & $4 d^{1} \mathrm{D}_{2}-5 p^{1} \mathrm{~F}_{3}^{\circ}$ & 3496.419 & 50 & 28592.51 & $5 p^{3} \mathrm{~F}_{3}^{\circ}-5 d^{3} \mathrm{G}_{4}$ \\
\hline 3227.507 & 15 & 30974.72 & $5 p^{3} \mathrm{D}_{1}^{\circ}-5 d^{3} \mathrm{~F}_{2}$ & 3500.57 & $1 H$ & 28558.6 & $5 p^{3} \mathrm{P}_{0}^{\circ}-5 d^{3} \mathrm{~S}_{1}$ \\
\hline 3229.92 & $\operatorname{tr}$ & 30951.6 & $5 p^{3} \mathrm{P}_{2}^{\circ}-5 d^{3} \mathrm{P}_{1}$ & 3504.10 & $1 H$ & 28529.8 & $4 d^{3} \mathrm{P}_{1}-5 p^{3} \mathrm{D}_{2}^{\circ}$ \\
\hline 3234.251 & 20 & 30910.14 & $5 p^{3} \mathrm{D}_{2}^{\circ}-5 d^{3} \mathrm{~F}_{3}$ & 3505.70 & 10 & 28516.8 & $5 p^{3} \mathrm{P}_{1}^{\circ}-5 d^{3} \mathrm{~S}_{1}$ \\
\hline 3241.460 & $40 H$ & 30841.40 & $5 p^{3} \mathrm{D}_{3}^{\circ}-5 d^{3} \mathrm{~F}_{4}$ & 3514.25 & $80 h$ & 28447.4 & $5 p^{3} \mathrm{~F}_{4}^{\circ}-5 d^{3} \mathrm{G}_{5}$ \\
\hline 3268.077 & $15 h$ & 30590.22 & $5 p^{3} \mathrm{P}_{2}^{\circ}-5 d^{3} \mathrm{P}_{2}$ & 3525.89 & $\operatorname{tr} H$ & 28353.5 & $5 p^{3} \mathrm{~F}_{3}^{\circ}-5 d^{3} \mathrm{G}_{3}$ \\
\hline 3274.931 & $5 H$ & 30526.20 & $4 d^{3} \mathrm{P}_{1}-5 p^{3} \mathrm{P}_{2}^{\circ}$ & 3545.98 & $\operatorname{tr} h$ & 28192.9 & $5 p^{3} \mathrm{~F}_{3}^{\circ}-5 d^{3} \mathrm{D}_{2}$ \\
\hline 3284.560 & 50 & 30436.71 & $5 p^{1} \mathrm{~F}_{3}^{\circ}-5 d^{1} \mathrm{G}_{4}$ & 3550.718 & 10 & 28155.27 & $5 p^{3} \mathrm{P}_{2}^{\circ}-5 d^{3} \mathrm{~S}_{1}$ \\
\hline 3294.259 & 40 & 30347.10 & $4 d^{3} \mathrm{P}_{2}-5 p^{3} \mathrm{P}_{2}^{\circ}$ & 3681.04 & 10 & 27158.5 & $5 p^{3} \mathrm{P}_{1}^{\circ}-5 d^{3} \mathrm{D}_{2}$ \\
\hline 3295.501 & 10 & 30335.66 & $5 p^{3} \mathrm{~F}_{2}^{\circ}-5 d^{3} \mathrm{~F}_{2}$ & 3691.236 & 15 & 27083.46 & $5 p^{3} \mathrm{P}_{2}^{\circ}-5 d^{3} \mathrm{D}_{3}$ \\
\hline 3298.371 & 20 & 30309.27 & $5 p^{3} \mathrm{~F}_{3}^{\circ}-5 d^{3} \mathrm{~F}_{3}$ & 3833.74 & $15 H$ & 26076.8 & $5 p^{1} \mathrm{P}_{1}^{\circ}-5 d^{1} \mathrm{P}_{1}$ \\
\hline 3303.719 & 5 & 30260.21 & $4 d^{3} \mathrm{P}_{0}-5 p^{3} \mathrm{P}_{1}^{\circ}$ & 4136.72 & $5 H$ & 24166.9 & $4 d^{1} \mathrm{~S}_{0}-5 p{ }^{1} \mathrm{P}_{1}^{\circ}$ \\
\hline 3314.175 & 2 & 30164.74 & $4 d^{3} \mathrm{P}_{1}-5 p^{3} \mathrm{P}_{1}^{\circ}$ & 4450.75 & $\operatorname{tr}$ & 22461.8 & $4 f^{3} \mathrm{D}_{3}^{\circ}-5 d^{3} \mathrm{P}_{2}$ \\
\hline 3318.788 & $5 h$ & 30122.82 & $4 d^{3} \mathrm{P}_{1}-5 p^{3} \mathrm{P}_{0}^{\circ}$ & 4479.195 & $2 h$ & 22319.18 & $4 f^{1} \mathrm{~F}_{3}^{\circ}-5 d^{1} \mathrm{D}_{2}$ \\
\hline 3328.527 & 30 & 30034.68 & $5 p^{3} \mathbf{F}_{4}^{\circ}-5 d^{3} \mathbf{F}_{4}$ & 4505.17 & $1 H$ & 22190.5 & $4 f^{3} \mathbf{F}_{3}^{\circ}-5 d^{3} \mathbf{F}_{2}$ \\
\hline 3333.986 & 15 & 29985.50 & $4 d^{3} \mathrm{P}_{2}-5 p^{3} \mathrm{P}_{1}^{\circ}$ & 4508.67 & $2 H$ & 22173.3 & $4 f^{3} \mathbf{F}_{4}^{\circ}-5 d^{3} \mathbf{F}_{4}$ \\
\hline 3334.79 & 60 & 29978.3 & $4 d^{1} \mathrm{G}_{4}-5 p^{1} \mathrm{~F}_{3}^{\circ}$ & 4518.58 & $8 H$ & 22124.6 & $4 f^{3} \mathrm{H}_{5}^{\circ}-5 d^{3} \mathrm{~F}_{4}$ \\
\hline 3385.336 & 1 & 29530.69 & $5 p^{1} \mathrm{~F}_{3}^{\circ}-5 d^{1} \mathrm{D}_{2}$ & 4565.63 & $\operatorname{tr} H$ & 21896.6 & $4 f^{3} \mathrm{G}_{3}^{\circ}-5 d^{3} \mathrm{~F}_{2}$ \\
\hline 3433.52 & $\operatorname{tr}$ & 29116.3 & $5 p^{3} \mathrm{D}_{1}^{\circ}-5 d^{3} \mathrm{D}_{2}$ & 4608.15 & $1 H$ & 21694.6 & $4 f^{3} \mathrm{G}_{5}^{\circ}-5 d^{3} \mathrm{~F}_{4}$ \\
\hline 3448.410 & 50 & 28990.57 & $5 p^{1} \mathrm{D}_{2}^{\circ}-5 d^{1} \mathrm{~F}_{3}$ & 4616.57 & $1 H$ & 21655.0 & $4 f^{3} \mathrm{G}_{4}^{\circ}-5 d^{3} \mathrm{~F}_{3}$ \\
\hline 3452.741 & $3 H$ & 28954.20 & $5 p^{3} \mathrm{D}_{2}^{\circ}-5 d^{3} \mathrm{G}_{3}$ & 4643.985 & $10 H$ & 21527.20 & $4 f^{1} \mathrm{H}_{5}^{\circ}-5 d^{1} \mathrm{G}_{4}$ \\
\hline 3455.325 & 15 & 28932.55 & $5 p^{1} \mathrm{P}_{1}^{\circ}-5 d^{1} \mathrm{D}_{2}$ & 4801.54 & $2 H$ & 20820.8 & $5 s{ }^{3} \mathrm{D}_{2}-5 p^{3} \mathrm{P}_{2}^{\circ}$ \\
\hline 3459.40 & $10 h$ & 28898.5 & $5 p^{3} \mathrm{D}_{1}^{\circ}-5 d^{3} \mathrm{D}_{1}$ & 4828.990 & $1 H$ & 20702.48 & $4 d^{1} \mathrm{~S}_{0}-5 p^{3} \mathrm{D}_{1}^{\circ}$ \\
\hline 3471.989 & 20 & 28793.69 & $5 p^{3} \mathrm{D}_{2}^{\circ}-5 d^{3} \mathrm{D}_{2}$ & 4841.26 & $20 h$ & 20650.0 & $5 s^{1} \mathrm{D}_{2}-5 p^{1} \mathrm{P}_{1}^{\circ}$ \\
\hline 3473.458 & 20 & 28781.51 & $5 p^{3} \mathrm{D}_{3}^{\circ}-5 d^{3} \mathrm{D}_{3}$ & 4845.21 & $3 h$ & 20633.2 & $5 s{ }^{3} \mathrm{D}_{1}-5 p^{3} \mathrm{P}_{1}^{\circ}$ \\
\hline 3487.63 & 30 & 28664.6 & $4 d^{1} \mathrm{D}_{2}-5 p^{1} \mathrm{D}_{2}^{\circ}$ & 4855.05 & $3 H$ & 20591.3 & $5 s^{3} \mathrm{D}_{1}-5 p^{3} \mathrm{P}_{0}^{\circ}$ \\
\hline
\end{tabular}


TABLE 3. Classified lines of V IV-Continued

\begin{tabular}{|c|c|c|c|c|c|c|c|}
\hline$\lambda$ air & Intensity & Wave number & Classification & $\lambda$ air & Intensity & Wave number & Classification \\
\hline 4886.36 & $20 h$ & 20459.4 & $5 s^{3} \mathrm{D}_{2}-5 p^{3} \mathrm{P}_{1}^{\circ}$ & 5175.950 & $30 h$ & 19314.75 & $5 s^{3} \mathrm{D}_{1}-5 p{ }^{3} \mathrm{~F}_{2}^{\circ}$ \\
\hline 4891.52 & $\operatorname{tr} h$ & 20437.8 & $4 f^{3} \mathrm{G}_{3}-5 d^{3} \mathrm{G}_{4}^{\circ}$ & 5222.93 & $5 H$ & 19141.0 & $5 s^{3} \mathrm{D}_{2}-5 p^{3} \mathrm{~F}_{2}^{\circ}$ \\
\hline 4899.56 & $\operatorname{tr} h$ & 20404.3 & $4 f^{3} \mathrm{H}_{4}^{\circ}-5 d^{3} \mathrm{D}_{3}$ & 5227.89 & $10 H$ & 19122.8 & $5 s^{3} \mathrm{D}_{2}-5 p^{3} \mathrm{D}_{2}^{\circ}$ \\
\hline 4906.280 & $40 h$ & 20376.35 & $5 s^{3} \mathrm{D}_{3}-5 p^{3} \mathrm{P}_{2}^{\circ}$ & 5262.164 & $50 h$ & 18998.3 & $5 s{ }^{3} \mathrm{D}_{1}-5 p^{3} \mathrm{D}_{2}^{\circ}$ \\
\hline 4913.083 & $10 h$ & 20348.14 & $4 f^{1} \mathrm{G}_{4}^{\circ}-5 d^{1} \mathrm{~F}_{3}$ & 5267.045 & $10 h$ & 18980.69 & $5 s^{3} \mathrm{D}_{3}-5 p{ }^{3} \mathrm{~F}_{3}^{\circ}$ \\
\hline 4916.94 & $2 H$ & 20332.2 & $4 f^{3} \mathrm{~F}_{3}^{\circ}-5 d^{3} \mathrm{D}_{2}$ & 5310.77 & $20 H$ & 18824.4 & $5 s^{3} \mathrm{D}_{2}-5 p^{3} \mathrm{D}_{2}^{\circ}$ \\
\hline 4954.408 & $1 h$ & 20178.41 & $4 f^{3} \mathrm{H}_{5}^{\circ}-5 d^{3} \mathrm{G}_{4}$ & 5352.320 & $60 h$ & 18678.29 & $5 s^{3} \mathrm{D}_{3}-5 p^{3} \mathrm{D}_{3}^{\circ}$ \\
\hline 4966.38 & $2 h$ & 20129.8 & $4 f^{3} \mathbf{G}_{3}^{\circ}-5 d^{3} \mathrm{~F}_{2}$ & 5353.090 & $25 H$ & 18675.60 & $5 s^{3} \mathrm{D}_{1}-5 p{ }^{3} \mathrm{D}_{1}^{\circ}$ \\
\hline 4970.348 & $3 h$ & 20113.70 & $4 f^{3} \mathbf{F}_{4}^{\circ}-5 d^{3} \mathrm{D}_{3}$ & 5387.210 & $3 h$ & 18557.32 & $4 f^{1} \mathrm{~F}_{3}^{\circ}-5 d^{1} \mathrm{~F}_{3}$ \\
\hline 4971.941 & $1 h$ & 20107.26 & $4 f^{3} \mathrm{G}_{5}^{\circ}-5 d^{3} \mathbf{G}_{5}$ & 5496.67 & $1 H$ & 18187.8 & $4 f^{3} \mathrm{D}_{3}^{\circ}-5 d^{1} \mathrm{~F}_{3}$ \\
\hline 4985.653 & $50 h$ & 20051.96 & $5 s^{1} \mathrm{D}_{2}-5 p^{1} \mathrm{~F}_{3}^{\circ}$ & 5509.19 & $30 H$ & 18146.4 & $5 s^{3} \mathrm{D}_{2}-5 p{ }^{1} \mathrm{D}_{2}^{\circ}$ \\
\hline 5035.460 & $10 h$ & 19853.62 & $4 f^{3} \mathrm{H}_{6}^{\circ}-5 d^{3} \mathrm{G}_{5}$ & 5520.63 & $2 H$ & 18108.9 & $5 s{ }^{1} \mathrm{D}_{2}-5 p^{3} \mathrm{~F}_{3}^{\circ}$ \\
\hline 5074.90 & $5 H$ & 19699.3 & $4 f^{3} \mathrm{G}_{4}^{\circ}-5 d^{3} \mathrm{G}_{3}$ & 5608.71 & $20 H$ & 17824.4 & $5 s^{1} \mathrm{D}_{2}-5 p{ }^{3} \mathrm{~F}_{2}^{\circ}$ \\
\hline 5130.78 & $50 H$ & 19484.8 & $5 s^{3} \mathrm{D}_{3}-5 p^{3} \mathrm{~F}_{4}^{\circ}$ & 5710.10 & $8 H$ & 17508.0 & $5 s^{1} \mathrm{D}_{2}-5 p^{3} \mathrm{D}_{2}^{\circ}$ \\
\hline 5146.502 & $40 h$ & 19425.26 & $5 s^{3} \mathrm{D}_{2}-5 p^{3} \mathrm{~F}_{3}^{\circ}$ & 5940.12 & $40 \mathrm{H}$ & 16830.0 & $5 s^{1} \mathrm{D}_{2}-5 p{ }^{1} \mathrm{D}_{2}^{\circ}$ \\
\hline
\end{tabular}

TABLE 4. Energy levels and limits of the ns series of $\mathrm{V}$ IV

\begin{tabular}{|c|c|c|c|c|c|c|}
\hline Electron & Desig. & Energy level & Term value & $n^{*}$ & Limit & I.P. \\
\hline $\begin{array}{l}4 s \\
5 s \\
6 s\end{array}$ & $\begin{array}{l}{ }^{3} \mathrm{D}_{3} \\
{ }^{3} \mathrm{D}_{3} \\
{ }^{3} \mathrm{D}_{3}\end{array}$ & $\begin{array}{r}96798.0 \\
236766.9 \\
292417.4\end{array}$ & $\begin{array}{r}280538.0 \\
140569.1 \\
84918.6\end{array}$ & $\begin{array}{l}2.50172 \\
3.53419 \\
4.54709\end{array}$ & 377336 & 376716 \\
\hline $\begin{array}{l}4 s \\
5 s \\
6 s\end{array}$ & $\begin{array}{l}{ }^{3} \mathrm{D}_{2} \\
{ }^{3} \mathrm{D}_{2} \\
{ }^{3} \mathrm{D}_{2}\end{array}$ & $\begin{array}{r}96412.1 \\
236322.4 \\
291918.1\end{array}$ & $\begin{array}{r}280353.9 \\
140443.6 \\
84847.9\end{array}$ & $\begin{array}{l}2.50254 \\
3.53577 \\
4.54898\end{array}$ & 376766 & 376766 \\
\hline $\begin{array}{l}4 s \\
5 s \\
6 s\end{array}$ & $\begin{array}{l}{ }^{3} \mathrm{D}_{1} \\
{ }^{3} \mathrm{D}_{1} \\
{ }^{3} \mathrm{D}_{1}\end{array}$ & $\begin{array}{r}96196.1 \\
236148.6 \\
291796.0\end{array}$ & $\begin{array}{r}280515.9 \\
140563.6 \\
84916.0\end{array}$ & $\begin{array}{l}2.50182 \\
3.53426 \\
4.54716\end{array}$ & 376712 & 376712 \\
\hline $\begin{array}{l}4 s \\
5 s \\
6 s\end{array}$ & $\begin{array}{l}{ }^{1} \mathrm{D}_{2} \\
{ }^{1} \mathrm{D}_{2} \\
{ }^{1} \mathrm{D}_{2}\end{array}$ & $\begin{array}{l}100200.7 \\
237638.8 \\
292766.7\end{array}$ & $\begin{array}{r}277134.3 \\
139696.2 \\
84568.3\end{array}$ & $\begin{array}{l}2.51704 \\
3.54522 \\
4.55651\end{array}$ & 377335 & 376715 \\
\hline
\end{tabular}




\section{4. $V$ v}

As a by-product of the V IV analysis, 8 lines of the fifth spectrum of vanadium were identified in the region 675 to $2200 \AA$. With the present measurements, the levels published in A.E.L. [2] giving combinations in this part of the spectrum have been corrected and the ${ }^{2} \mathrm{D}$ term of the $4 d$ configuration has been found. The new values based on the assumption that $4 p^{2} \mathrm{P}_{11 / 2}^{\circ}$ $=207617.0 \mathrm{~cm}^{-1}$ is correct, are the following:
New levels of $\mathrm{V} \mathrm{V}$

\begin{tabular}{c|c|c|c}
\hline \hline Config. & Desig. & $J$ & Level \\
\hline $4 s$ & ${ }^{2} \mathrm{~S}$ & $0^{\frac{1}{2}}$ & 148100.1 \\
$4 p$ & ${ }^{2} \mathrm{P}^{\circ}$ & $0^{\frac{1}{2}}$ & 206350.6 \\
$4 d$ & ${ }^{2} \mathrm{D}$ & $1^{\frac{1}{2}}$ & 293859.5 \\
& & $2 \frac{1}{2}$ & 294004.3 \\
$5 s$ & ${ }^{2} \mathrm{~S}$ & $0^{\frac{1}{2}}$ & 328173.5 \\
$4 f$ & ${ }^{2} \mathrm{~F}^{\circ}$ & $3 \frac{1}{2}$ & 349210.6 \\
\hline
\end{tabular}

the observed combinations among them are as follows:

Newly classified lines of $\mathrm{V} \mathbf{v}$

\begin{tabular}{c|c|c|c}
\hline \hline$\lambda$ & Int. & Wave number & Classification \\
\hline 820.866 & 15 & 121822.5 & $4 p^{2} \mathrm{P}^{011 / 2}-5 s^{2} \mathrm{~S}_{01 / 2}$ \\
829.483 & 20 & 120557.0 & $4 p^{2} \mathrm{P}_{11 / 2}^{\circ}-5 s^{2} \mathrm{~S}_{01 / 2}$ \\
1142.741 & 40 & 87508.9 & $4 p^{2} \mathrm{P}_{01 / 2}^{\circ}-4 d^{2} \mathrm{D}_{11 / 2}$ \\
1157.577 & 50 & 86387.3 & $4 p^{2} \mathrm{P}_{11 / 2}^{\circ}-4 d^{2} \mathrm{D}_{21 / 2}^{\circ}$ \\
1159.520 & 10 & 86242.6 & $4 p^{2} \mathrm{P}_{11 / 2}^{\circ}-4 d^{2} \mathrm{D}_{11 / 2}^{\circ}$ \\
1680.199 & 100 & 59516.9 & $4 s^{2} \mathrm{~S}_{01 / 2}-4 p^{2} \mathrm{P}_{11 / 2}^{\circ}$ \\
1716.722 & 50 & 58250.5 & $4 s^{2} \mathrm{~S}_{01 / 2}-4 p^{2} \mathrm{P}_{01 / 2}^{\circ}$ \\
1811.388 & $40 h$ & 55206.3 & $4 d^{2} \mathrm{D}_{21 / 2}^{\circ}-4 f^{2} \mathrm{~F}_{31 / 2}^{\circ}$ \\
\hline
\end{tabular}

The author is indebted to A. G. Shenstone for the many facilities placed at her disposal to carry out part of the experimental work on which this paper is based, and to R. Velasco for the use of his hollow cathode spectrograms and for many helpful discussions during the course of this investigation. Thanks are also due to Mrs. Charlotte Moore-Sitterly (NBS) for her kind cooperation in the correction of the English manuscript and during the editorial stages of this paper.

(Paper 72A4-503)

\section{References}

[1] White, H. E., Phys. Rev. 33, 538 (1929).

[2] Edlén, B., See C. E. Moore, Atomic Energy Levels, NBS Circ. 467, Vol. I (1949).

[3] Shenstone, A. G., J. Opt. Soc. Am. 44, 749 (1954).

[4] Iglesias, L. and Velasco, R., An. Soc. Esp. Fis. Quim. 54A, 83 (1958).

[5] Joint Commission for Spectroscopy, J. Opt. Soc. Am. 43, 410 (1953).

[6] Shadmi, Y., J. Opt. Soc. Am. 56, 647 (1966).

[7] Edlén, B., unpublished material (1967).

[8] Edlén, B., See C. E. Moore, Atomic Energy Levels, NBS Circ. 467, Vol. II (1952). 